\title{
Modeling Inter-Individual Variability in Sugar Beet Populations
}

\author{
Philippe de Reffye ${ }^{* \dagger}$, Sébastien Lemaire ${ }^{\ddagger *}$, Nitish Srivastava ${ }^{\S} \uparrow$, Fabienne Maupas ${ }^{\ddagger}$, Paul-Henry Cournède $\boldsymbol{q}^{\dagger}$ \\ ${ }^{*}$ CIRAD, UMR AMAP, Montpellier, France \\ ${ }^{\dagger}$ INRIA Saclay Ille-de-France, EPI Digiplante, Orsay, France \\ ¥ Institut Technique de la Betterave sucrière, Paris, France \\ $\S$ IIT Kanpur, Department of Computer Science, Kanpur, India \\ ${ }^{\top}$ Ecole Centrale Paris, Laboratory of Applied Maths and Systems - Châtenay-Malabry, France \\ Corresponding author: paul-henry.cournede@ecp.fr
}

\begin{abstract}
Modeling heterogeneity in field crops is a key issue for a better characterization of field production. This paper presents some experimental data on sugar beet illustrating this heterogeneity. Several sources of individual variability within plant populations are identified: namely, initial condition (seed biomass, emergence delay), genetic variability (including phyllochron) and environment (including spacing and competition). A mathematical framework is introduced to integrate the different sources of variability in plant growth models. It is based on the classical method of Taylor Series Expansion, which allows the propagation of uncertainty in the dynamic system of growth and the computation of the approximate means and standard deviations of the model outputs. The method is applied to the GreenLab model of plant growth and more specifically to sugar beet. It opens perspectives in order to assess the different sources of variability in plant populations and estimate their parameters from experimental data. However important issues like optimization of data collection and system identifiability have to be resolved first, since the uncertainty effects may be mixed in an inextricable way or may necessitate a too huge amount of experimental data for their estimation.
\end{abstract}

\section{Introduction}

Modeling crops as communities of individual plants has brought new insights to understand and describe crop production [1], and can be seen as a complementary approach to the more classical crop / process-based models describing ecophysiological processes per field unit surface area [2]. The literature on individual-based plant growth models is now abundant (we refer to [3] introducing some of the most recent advances). Such models are usually seen as integrative systems, allowing a very detailed description of ecophysiological processes, at different biological scales [4].

So far, few of them aim at a real predictive capacity and are calibrated from experimental data [5], [6], [7]. Likewise, the extrapolation of individual-based models to the field scale is still at its early stages. It mostly concerns competition for light, by considering radiosity models to compute light interception [8] or empirical functions to describe interactions between neighbours [9]. The latter approach based on the GreenLab model of plant growth has led to model calibration at the population level in different density conditions for maize [10], tomato [11] or sugar beet [12]. The calibration process is based on an average individual plant. Such result is an improvement compared to classical crop models giving the compartment biomasses per unit surface area, since beside the compartments which are also simulated, the average plant is described at the organ level. However, owing to the strong variability among individuals, only predicting the average plant growth is also restrictive, it only gives a partial characterization of field production.

The objective of this paper is to identify the different sources of variability in sugar beet growth and propose a mathematical framework based on the theory of uncertainty propagation ([13], [14]) to integrate this variability in individual plant growth models. This way, we are able to describe the statistical distribution of individual productions and architectures in a population of plants. Such method can be seen as a first step towards the design of population models.

Section 2 introduces some experimental results for sugar beet underlining the strong heterogeneity within a population. The different sources of variability are also identified. In Section 3, we recall some basic concepts of the Taylor Series Expansion (TSE) method to propagate uncertainty in dynamic system and show how to apply it to the GreenLab model of plant growth. Some computations are performed to compare the approximations of means and standard deviations of model outputs to Monte Carlo simulations. Finally, the perspectives of such method for parametric estimation or for applications to other crops are discussed. 

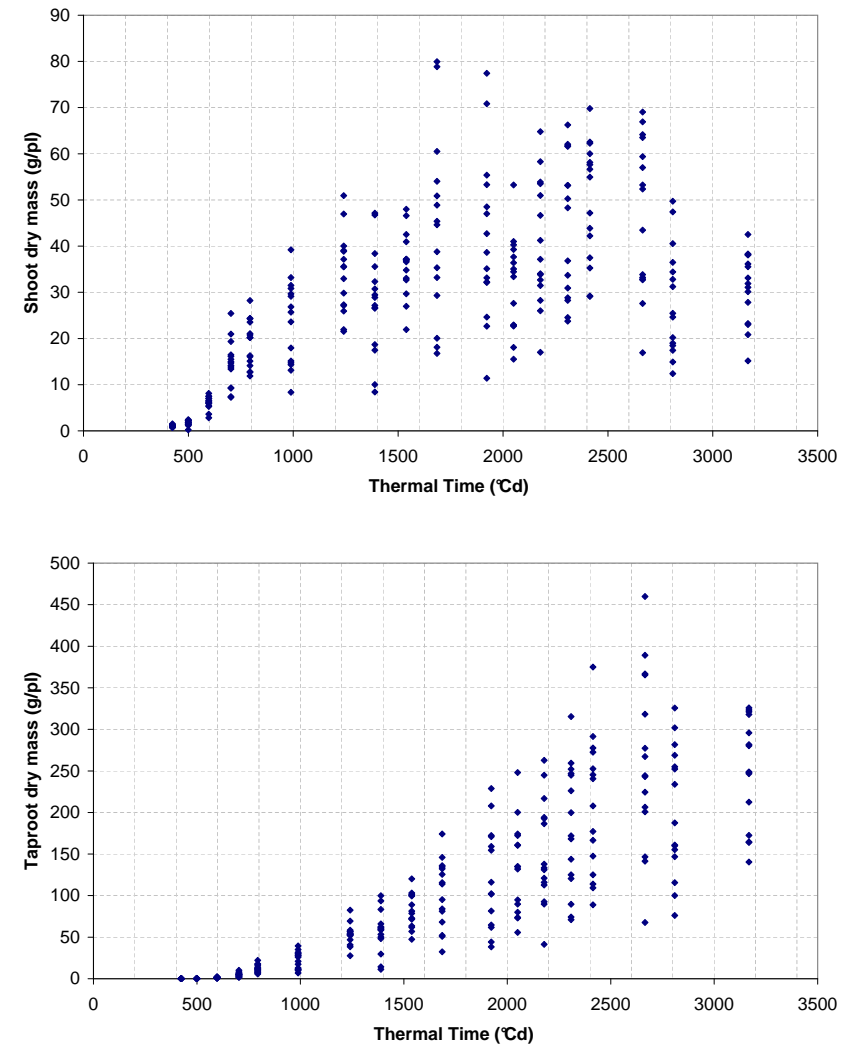

Figure 1. Individual variability in sugar beet fields: shoot and root dry masses versus thermal time for all measured individuals.

\section{Inter-individual variability in sugar beet populations}

Field experiments were conducted in 2006 by the ITB (Instut Technique de la Betterave sucrière) to investigate the source-sink dynamics of sugar beet growth with the GreenLab model (see [15] for more details on the experimental work). The final population was estimated to be 9.6 plants per $m^{2}$. The thermal time, corresponding to the accumulation of daily temperature above a base temperature $\left(0^{\circ} \mathrm{C}\right)$ was calculated from emergence. Destructive biomass measurements were carried out at eighteen different stages during the growing period. At each date, 15 individual plants were selected and the dry masses were measured at the level of organ compartments: total dry mass of blades, that of petioles and that of root. The final stage of measurements corresponds to harvest. The results are shown in Figure 1 and reveal a very strong variability among individuals.

For mono-specific crops, 3 sources of individual variability are identified [16]: first, growth initial conditions, which are given by the seed biomass and emergence delay; second

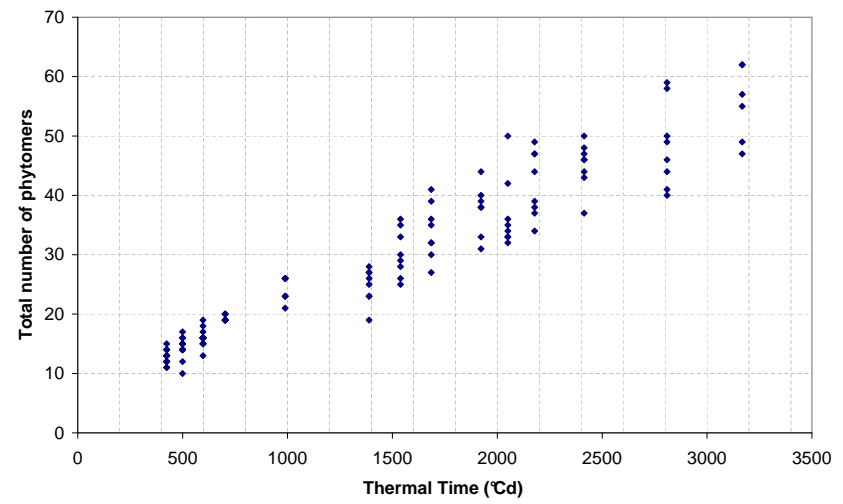

Figure 2. Inter-individual variability of sugar beet phyllochron: number of phytomers versus thermal time for all measured individuals.

the environmental conditions, which may strongly vary in a field, from one plant to another, (including spacing with neighbours); finally genetic variability: some species, like sugar beet, are not pure-bred [17] and it may result in variable responses to growth environmental conditions among individuals.

A good illustration of this genetic variability in sugar beet is given by the phyllochron (that is to say the thermal time elapsing between the appearance of two successive leaves). For most crops, the phyllochron usually shows a good range of stability, even though it may be strongly affected by environmental factors, see [18] for a short review on the main results for different plants. In sugar beet populations, [12] found that the mean phyllochron is extremely stable (during the first stage of growth) among seasons and for different density, hydric and nitrogen conditions. However, if the mean is stable, the differences among individuals are very important as shown by Figure 2. Such strong variability between individuals within a cultivar was reported by other authors (see for exemple [19]). The key issue is thus to set a proper framework to integrate the different sources of variability in individual plant growth models. It is examined in the next section.

\section{Propagation of uncertainty in plant growth models}

In forest ecosystem modeling, some results were obtained to assess the effects of individual variability for static allometry models ([20], [21]) in the statistical frame of hierarchical models. The problem is more complex for dynamic system of plant growth: the different sources of variability given above may be seen as uncertain inputs of the dynamic system, and the variability in system outputs results from 
the propagation of probabilistic uncertainty. This issue has already been studied in the context of crop models [22]. The proposed method to study the output distribution is based on Monte Carlo sampling, which might be restrictive regarding the computing cost when model inversion is involved (for parameter estimation at population level for example) or to solve optimization problems. There exist several other methods widely used in physics [13], [23] that overcome this difficulty. Here, we recall a method to compute uncertainty propagation based on Taylor series expansion, and we apply it in the context of the GreenLab model of plant growth.

\subsection{A general method based on Taylor series ex- pansion}

We recall here the basic concepts of the classical Taylor Series Expansion (TSE) method (see for exemple [24] for more details).

Let $x$ be a random vector in $\mathbb{R}^{d}$ with mean $\bar{x}$ and covariance matrix $P$. Let $g: \mathbb{R}^{d} \rightarrow \mathbb{R}$ a transformation of $x, y=g(x)$. Statistics on $y$ can be obtained by Monte Carlo simulations [22]. However, the evaluation cost of $g$ can be prohibitive for such simulations. A classical approximation of the moments of $g(x)$ can be obtained by considering the multivariate Taylor series expansion of $g(x)$ about $\bar{x}$ [13]. If we consider $x$ as the sum of $\bar{x}$ and a zero-mean disturbance $\Delta x$, we have for all $n>0$ :

$g(x)=g(\bar{x})+D_{\Delta x} g(\bar{x})+\frac{D_{\Delta x}^{2} g(\bar{x})}{2 !}+\cdots+\frac{D_{\Delta x}^{n} g(\bar{x})}{n !}+o\left(\|\Delta x\|^{n}\right)$

where $D_{\Delta x}$ is the differential operator corresponding to the perturbation $\Delta x$

$$
D_{\Delta x}=\sum_{i=1}^{d} \Delta x_{i} \frac{\partial}{\partial x_{i}}
$$

such that

$$
D_{\Delta x} g(\bar{x})=\sum_{i=1}^{d} \Delta x_{i} \frac{\partial g}{\partial x_{i}}(\bar{x})
$$

and

$$
D_{\Delta x}^{i} g(\bar{x})=\left[\left(\sum_{i=1}^{d} \Delta x_{i} \frac{\partial}{\partial x_{i}}\right)^{i} g\right](\bar{x}) .
$$

We can approximate the expectation and variance of $g(x)$, respectively $E[g(x)]$ and $V[g(x)]$ thanks to the Taylor series (1). Since $\Delta x$ is zero-mean variable, its first moment is zero, and we have:

$$
\begin{aligned}
E[g(x)]= & g(\bar{x})+\frac{1}{2} \sum_{i, j} \frac{\partial^{2} g}{\partial x_{i} \partial x_{j}}(\bar{x}) E\left[\Delta x_{i} \Delta x_{j}\right] \\
& +E\left[\frac{1}{3 !} D_{\Delta x}^{3} g(\bar{x})+\cdots\right],
\end{aligned}
$$

and we finally have the approximation, with an error at the third order:

$$
E[g(x)] \approx g(\bar{x})+\frac{1}{2} \sum_{i, j} \frac{\partial^{2} g}{\partial x_{i} \partial x_{j}}(\bar{x}) P_{i j} .
$$

For the variance, we have:

$$
\operatorname{Var}[g(x)]=E\left[(g(x)-E[g(x)])^{2}\right],
$$

with

$$
\begin{gathered}
g(x)-E[g(x)]=D_{\Delta x} g(\bar{x})+\frac{D_{\Delta x}^{2} g(\bar{x})}{2 !}+\left(\frac{D_{\Delta x}^{3} g(\bar{x})}{3 !}+\cdots\right) \\
-\frac{1}{2} \sum_{i, j} \frac{\partial g}{\partial x_{i}}(\bar{x}) \frac{\partial g}{\partial x_{i}}(\bar{x}) P_{i j}-E\left[\frac{D_{\Delta x}^{3} g(\bar{x})}{3 !}+\cdots\right]
\end{gathered}
$$

and we have the approximation with an error at the third order given by:

$$
\operatorname{Var}[g(x)] \approx E\left[\left(D_{\Delta x} g(\bar{x})\right)^{2}\right]
$$

and thus

$$
\operatorname{Var}[g(x)] \approx \sum_{i, j} \frac{\partial g}{\partial x_{i}}(\bar{x}) \frac{\partial g}{\partial x_{j}}(\bar{x}) P_{i j} .
$$

Remark 1: Since the standard deviation is the square root of the variance and is comparable to $\bar{x}$ (same unit), the order of the approximation for the variance in 3 may prove unsufficient. We shall consider increasing the order of the approximation, but it requires the knowledge of the moments of higer orders for the distribution of $x$.

Remark 2: If $g$ is not scalar and takes its value in $\mathbb{R}^{m}$, the same principle applies for each component function $g_{k}$, $1 \leq k \leq m$. The only difference is that we also need to determine an approximation of all $\operatorname{Cov}\left(g_{k}(x), g_{l}(x)\right)$ given by:

$$
\operatorname{Cov}\left(g_{k}(x), g_{l}(x)\right) \approx \sum_{i, j} \frac{\partial g_{k}}{\partial x_{i}}(\bar{x}) \frac{\partial g_{l}}{\partial x_{j}}(\bar{x}) P_{i j}
$$

\subsection{Application to the GreenLab equation of plant growth}

In this section, we first show how to characterize the production distribution in a plant population on a simple example. We suppose that the main source of variability in the population is the seed mass, which is given by a Gaussian law $\mathcal{N}\left(u_{0}, \sigma_{0}\right)$, and we want to deduce the distribution of the total biomass produced by each individual in the population at a given time $T$. In GreenLab, the time step is chosen to coincide with the growth cycle, based on phytomer appearance. $T$ growth cycles thus correspond to $T$ phyllochrons.

We recall the classical GreenLab equation giving $Q^{t}$, plant biomass production at growth cycle $t$ related to Beer's law [9]:

$$
Q^{t}=E^{t} \mu S_{p}\left(1-\exp \left(-k \frac{S^{t}}{S_{p}}\right)\right)
$$

where $E^{t}$ is the environmental input at growth cycle $t, \mu$ is plant efficiency, $S_{p}$ is a characteristic surface related to plant 
competition, $k$ is Beer's coefficient of light interception and $S^{t}$ is the photosynthetic leaf surface area at growth cycle $t$. Since $S^{t}$ is the result of successive biomass allocation to leaves, $S^{t}$ is a function of biomass production at the previous cycles $Q^{t-1}, Q^{t-2}, \ldots$ We get (see [25]):

$$
S^{t}=-\frac{1}{e} \sum_{k=t-T_{b}}^{t-1} \frac{Q^{k}}{D^{k}}\left(\sum_{j=0}^{k+T_{b}-t} N_{b}^{k}(j) p_{b}^{k}(j)\right)
$$

with $N_{b}^{k}(j)$, the number of leaves of chronological age $j$ at growth cycle $k, p_{b}^{k}(j)$ their corresponding sinks, $T_{b}$ leaf life span, $e$ specific blade mass (supposed constant) and $D^{k}$ plant demand at growth cycle $k$ computed as the sum of all living sinks at growth cycle $k$.

If there is no retroaction of growth on plant organogenesis, the functions giving the numbers of organs as well as the demand are given. The environmental function $E^{t}$ is also supposed given. Therefore, substituting Eq. (5) into Eq. (4) yields a recurrent equation of order $T_{b}$ for the sequence $\left(Q^{t}\right)_{t}$.

For a direct application of the method presented in Section 3.1 , we first rewrite the dynamic system of growth. Let us define the state vector $X^{t} \in \mathbb{R}^{T+1}$ as :

$$
X^{t}=\left(\begin{array}{c}
Q^{0} \\
Q^{1} \\
\vdots \\
Q^{t} \\
0 \\
0 \\
\vdots \\
0
\end{array}\right)
$$

The system evolution equation is thus written for all $n \leq$ $T-1$ :

$$
X^{t+1}=F^{t}\left(X^{t}\right)
$$

with $\left(F^{t}\right)_{i}, 1 \leq i \leq T+1$, the components of $F^{t}$ given by:

$$
\begin{gathered}
F_{i}^{t}(X)=(X)_{i} \quad \text { for } 1 \leq i \leq t+1, \\
F_{t+2}^{t}(X)=E^{t+1} \mu S_{p}\left(1-\exp \left(\frac{k S^{t+1}}{S_{p}}\right)\right)
\end{gathered}
$$

with

$$
S^{t+1}=\frac{1}{e} \sum_{k=t-T_{b}+1}^{t} \frac{X_{k+1}}{N^{k} \cdot p^{k}}\left(\sum_{j=0}^{k+T_{b}-t-1} N_{b}^{k}(j) p_{b}^{k}(j)\right) .
$$

and

$$
\left(F^{t}\right)_{i}(X)=0 \text { for } t+2<i \leq T+1 .
$$

We can apply the methodology introduced in Section 3.1 to propagate the uncertainty in the dynamic system in the form of Equation (7). In this way, we can determine recursively an approximation of the distribution moments of $X^{T}$.
Note that partial derivatives can be computed analytically. The initial condition is given by:

$$
\overline{X^{0}}=\left(\begin{array}{c}
u^{0} \\
0 \\
\vdots \\
0
\end{array}\right) \text { and } \operatorname{Cov}\left(X^{0}\right)=\left(\begin{array}{cccc}
\sigma_{0}^{2} & 0 & \cdots & 0 \\
0 & 0 & \cdots & 0 \\
\vdots & \ddots & \ddots & \vdots \\
0 & \cdots & 0 & 0
\end{array}\right)
$$

and if we suppose known $\overline{X^{t}}$ and $\operatorname{Cov}\left(X^{t}\right)$, we deduce $\overline{X^{t+1}}$ and $\operatorname{Cov}\left(X^{t+1}\right)$ as follows.

- For the mean:

- if $1 \leq i \leq T+1, i \neq t+2$ :

$$
\overline{X_{i}^{t+1}}=\overline{X_{i}^{t}}
$$

- and

$\overline{X_{t+2}^{t+1}}=F_{t+2}^{t}\left(\overline{X^{t}}\right)+\frac{1}{2} \sum_{i, j} \frac{\partial^{2} F_{t+2}^{t}}{\partial X_{i} \partial X_{j}}\left(\overline{X^{t}}\right) \operatorname{Cov}\left(X^{t}\right)_{i j}$.

- For the covariance:

- for $1 \leq i \leq t+1,1 \leq j \leq t+1$ :

$$
\operatorname{Cov}\left(X^{t+1}\right)_{i j}=\operatorname{Cov}\left(X_{i}^{t+1}, X_{j}^{t+1}\right)_{i j}=\operatorname{Cov}\left(X^{t}\right)_{i j}
$$

- for $i>t+2$ ou $j>t+2$ :

$$
\operatorname{Cov}\left(X^{t+1}\right)_{i j}=0
$$

- finally, for $j<t+2$ :

$\operatorname{Cov}\left(X_{t+2}^{t+1}, X_{j}^{t+1}\right)=\sum_{k, l} \frac{\partial F_{t+2}^{t}}{\partial X_{k}}\left(\overline{X^{t}}\right) \frac{\partial F_{j}^{t}}{\partial X_{l}}\left(\overline{X^{t}}\right) \operatorname{Cov}\left(X^{t}\right)_{k l}$, and

$$
\operatorname{Cov}\left(X_{j}^{t+1}, X_{t+2}^{t+1}\right)=\operatorname{Cov}\left(X_{t+2}^{t+1}, X_{j}^{t+1}\right) .
$$

It is also possible to choose an approximation of higer order.

Finally, we obtain the approximations of the mean and variance of plant biomass production at any growth cycle $t$, since:

$$
\overline{Q^{t}}=\overline{X_{t+1}^{t}} \text { and } \operatorname{Var}\left(Q^{t}\right)=\operatorname{Cov}\left(X_{t+1}^{t}, X_{t+1}^{t}\right) .
$$

A numerical application of these results is given in Figure 3, in which are shown the evolutions of the mean and standard deviation of biomass production with the growth cycle. For the gaussian distribution of seed biomass, we have chosen standard deviations corresponding to $10 \%$ and $20 \%$ of the mean seed mass. There is a very good agreement between the Monte Carlo simulations and the approximations given by the theoretical formula (12) and (13), which seems to indicate a good range of validity for the TSE method. It is interesting to note that the standard deviation of the biomass production reaches a peak and then decreases to zero. The reason is the saturation of biomass production due to Beer's law: after some time, even the plants with the smallest seeds will reach the limit production in our example.

All the variables of interest (organ masses, compartment 
(a)

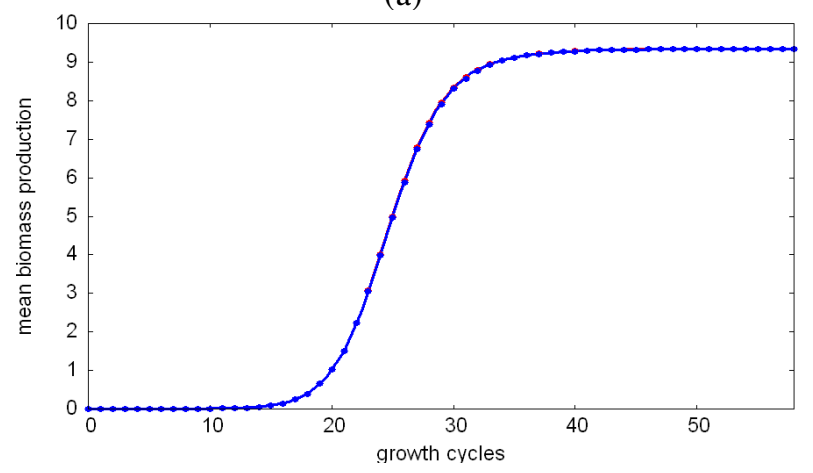

(b)

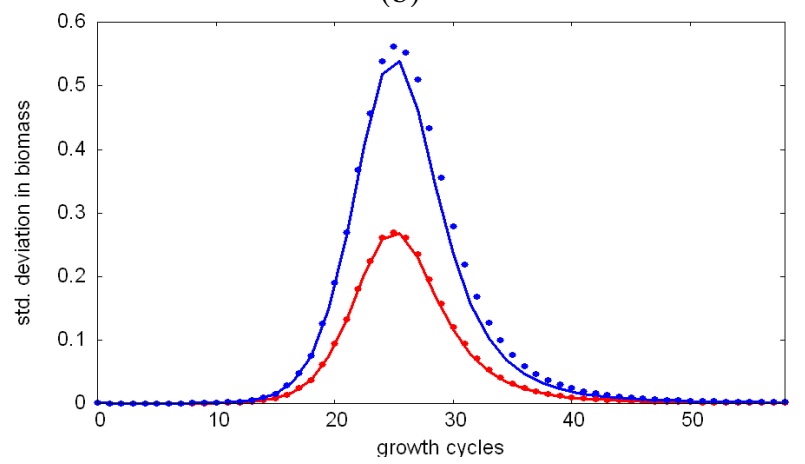

Figure 3. Effects of the variance in seed biomass: (a) the red line gives the evolution of the approximation of mean biomass production for $\sigma_{0}=0.1$ and the blue line for $\sigma_{0}=0.2$ (both lines are superposed); the dots are the corresponding Monte Carlo results for $10^{4}$ simulations. (b) The red line gives the evolution of the standard deviation of biomass production for $\sigma_{0}=0.1$ and the blue line for $\sigma_{0}=0.2$; the dots are the corresponding Monte Carlo results for $10^{4}$ simulations.

masses...) can be obtained as linear combinations of $\left(Q^{t}\right)_{t}$, therefore it is straightforward to get their approximate means and variances. For example, for the total plant biomass $M(T)$, we have:

$$
\overline{M(T)}=\sum_{i=0}^{T+1} \overline{X_{i}^{T}},
$$

with variance

$$
\operatorname{Var}(M(T))=\sum_{k, l} \operatorname{Cov}\left(X^{T}\right)_{k l} .
$$

We now turn to a more complex computation, which we perform for conditions close to those of sugar beet population. We use the parameters estimated from 2006 experimental data and given in [15] and we choose to consider 4 soures of variability, all gaussian: seed biomass, local environment $E^{t}$, competition effects due to uneven spacing, which is characterized in the GreenLab model by the parameter $S_{p}$, and phyllochron. The emergence delay is not taken into account since the experimental observations (a)

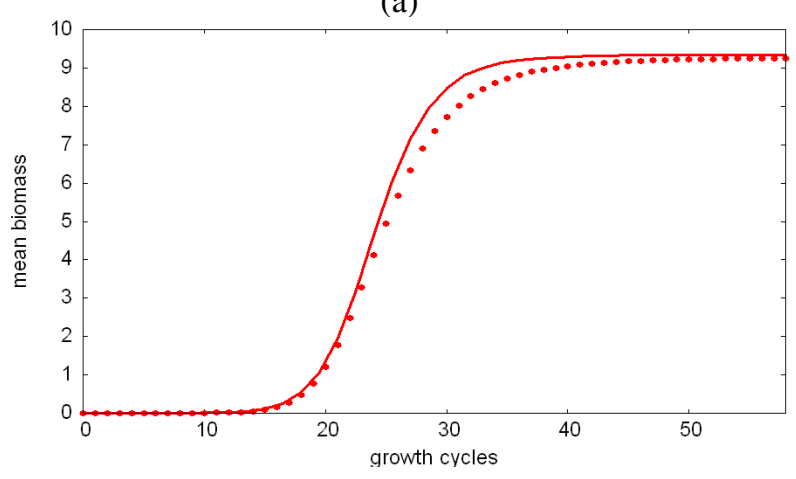

(b)

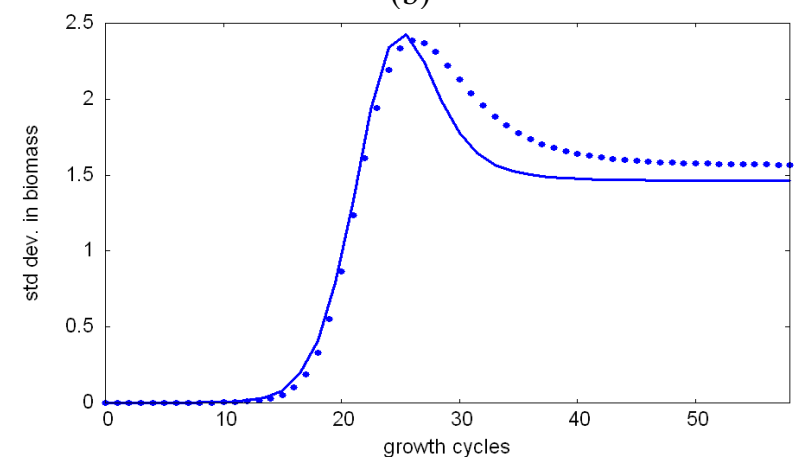

Figure 4. Effects of the variances in seed biomass $u^{0}$, environment $E, S_{p}$ and phyllochron $P$ : (a) the red line gives the evolution of the mean biomass production computed with the approximation formula, the dots are the corresponding Monte Carlo results for $10^{4}$ simulations; (b) the blue line gives the evolution of standard deviation in biomass production computed with the approximation formula, the dots are the corresponding Monte Carlo results for $10^{4}$ simulations.

showed a delay inferior to the phyllochron.

The formula giving the approximation formula for the mean and variance of biomass production are not presented in details but derive from the multivariate versions of Equations (2) and (3). The computations are heavy but quite straightforward.

The results are shown in Figure 4: the standard deviation of seed biomass $Q^{0}$ corresponds to $20 \%$ of the mean mass; the environment $E^{t}$ is chosen constant for an individual plant, but with a standard deviation of $10 \%$ of its mean value in the population; $S_{p}$ has a standard deviation of $10 \%$ of its mean value in the population; and the standard deviation of the phyllochron is also $10 \%$ of its mean value. The approximation formula are compared to Monte Carlo simulation results. Small differences appear, but remain acceptable. We also compute the correlation coefficients between the growth rate $Q^{t}$ and the variation factors $u_{0}$, $E, S_{p}$ and $P$ (Phyllochron) during plant growth. The results are given in Figure 5. It is interesting to see that, in keeping with our remark on the previous exemple, the effect of seed 


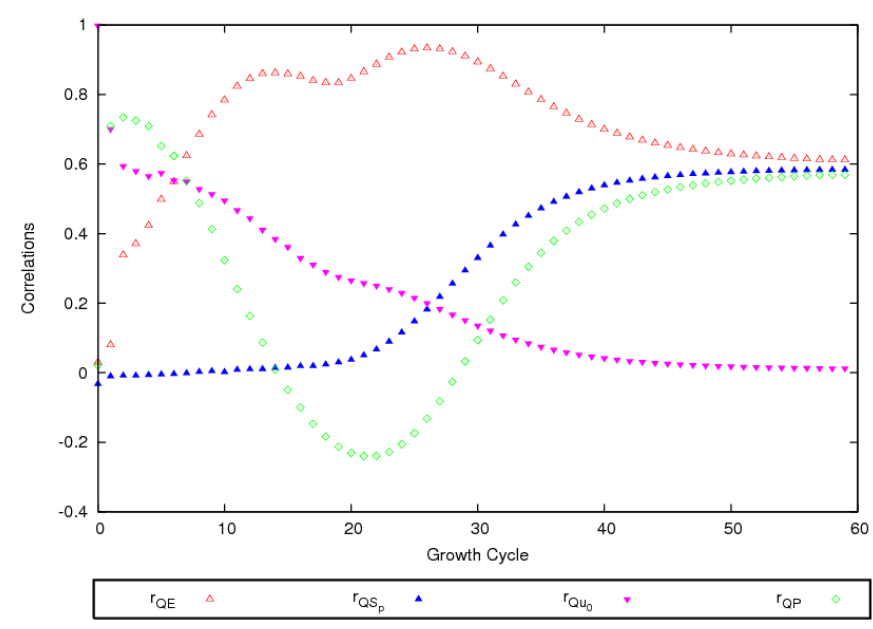

Figure 5. Evolution of the correlations between $Q^{t}$ and the different sources of variability: seed biomass $u_{0}\left(r_{Q u_{0}}\right)$, environment $E^{t}\left(r_{Q E}\right)$, local competition $S_{p}$ $\left(r_{Q S_{p}}\right)$ and phyllochron $P\left(r_{Q P}\right)$.

variation decreases to zero, while the variation of $S_{p}$ has no effect at the beginning and increases when the leaf area index begins to saturate. The effect of the environment variation is important during the whole growth as expected. The evolution of the correlation between $Q^{t}$ and the phyllochron $\left(r_{Q P}\right)$ is interesting: the plant takes advantage of a longer phyllochron in the early and late stages of growth, while the influence is less (or even negative) in the mid-stage. It can be explained by the evolution of the ratio of the competing sinks at the beginning of growth: blade sink variation function increases quicker than that of petiole. Thus, having older organs (which is a consequence of a longer phyllochron) favour the development of leaf surface area and consequently $Q^{t}$. The influence decreases when biomass production saturates, but when senescence starts, the effects of the early differences are visible again.

\section{Discussion}

Experimental observations of sugar beet populations have revealed a very strong inter-individual variability. In order to take into account this variability and to improve the statistical description of the population, we have proposed a framework to integrate different sources of variability in an individual-based crop model, GreenLab. The classical Taylor series expansion method is used to propagate uncertainty in the dynamic system of plant growth. Good approximations of the mean and standard deviations of the variables of interest (organ or compartment masses, biomass production...) can be computed.

When the sources of variability are well characterized, the method is simply a way to bypass Monte Carlo simulations.
A more interesting application is when the sources of variability are identified but not fully characterized: the method is well-adapted to model inversion (contrary to Monte Carlo simulations) and to the estimation of uncertainty distribution parameters (mean, standard deviation). If the experimental sampling is important enough, the experimental target to fit is composed of both mean values and standard deviations and such outputs are provided by the model. Some preliminary fitting experiments, not presented in this paper, were performed and gave some indications. They seem to work well when one or two sources of variability are considered, but when too many sources are considered together, some identifiability problems seem to occur: it is difficult to distinguish between all the variance effects. Some complementary studies are needed to fully assess the identification method. It is interesting to note that the computation have been done without using the Gaussian hypothesis. Only the means and standard deviations of the sources of variability are considered. However, for strongly non-linear systems, we may need to increase the approximation order, which would require the knowledge of higher order moments (which are easily obtained, for example, under the Gaussian hypothesis). Likewise, when the levels of variations become important, we have to study in more details the quality of the approximation. We have seen in Figure 4 that approximations given by Equations (2) and (3) begin to show some defaults when several sources of variability are considered together at reasonable levels corresponding to variation coefficients of $10 \%$. It is always possible to increase the approximation order (see [24]), but the formulas become tedious. Resorting to symbolic computation software may prove of great help to perform differentiation.

Finally, if this method was illustrated on sugar beet, it seems potentially applicable to a wide variety of crops and its interest is crucial for heterogeneous crops, e.g. rapeseed. From a purely theoretical point of view, there is no limit to the method, as long as the underlying model is properly defined. However, the computation might prove very complex, as well as the data collection, since an important amount of individuals have to be measured to get proper information on variances within population. Moreover, the confrontation to experimental data might raise the issue of system identifiability since the variability effects might be too strongly inter-connected.

The next step is thus to study in details the parametric estimation from experimental data derived from this modeling approach taking into account inter-individual variability within plant populations.

\section{Acknowledgment}

The authors would like to thank the two anonymous reviewers for helping improving the clarity of the manuscript. N. Srivastava is supported by the Summer Undergraduate 
Research Grant for Excellence (SURGE) between IIT Kanpur and Centrale Paris.

\section{References}

[1] C. Fournier and B. Andrieu, "A 3d architectural and processbased model of maize development," Annals of Botany, no. 81, pp. 233-250, 1998.

[2] P. de Reffye, E. Heuvelink, D. Barthélémy, and P.-H. Cournède, "Plant growth models," in Ecological Models. Vol. 4 of Encyclopedia of Ecology (5 volumes), S. Jorgensen and B. Fath, Eds. Elsevier (Oxford), 2008, pp. 2824-2837.

[3] J. Vos, L. Marcelis, P. de Visser, P. Struik, and J. Evers, Functional-structural plant modelling in crop production. Springer, 2007.

[4] G. Buck-Sorlin, R. Hemmerling, O. Kniemeyer, B. Burema, and W. Kurth, "A rule-based model of barley morphogenesis, with special respect to shading and gibberellic acid signal transduction," Annals of Botany, vol. 101, no. 8, 2008.

[5] Y. Guo, Y. Ma, Z. Zhan, B. Li, M. Dingkuhn, D. Luquet, and P. de Reffye, "Parameter optimization and field validation of the functional-structural model greenlab for maize," Annals of Botany, vol. 97, pp. 217-230, 2006.

[6] D. Luquet, M. Dingkuhn, H. Kim, L. Tambour, and A. Clément-Vidal, "Ecomeristem, a model of morphogenesis and competition among sinks in rice. 1. concept, validation and sensitivity analysis," Functional Plant Biology, vol. 33, no. 4, pp. 309-323, 2007.

[7] P. Wernecke, J. Müller, T. Dornbusch, A. Wernecke, and W. Diepenbrock, "The virtual crop-modelling system 'vica' specified for barley," in Functional-structural plant modelling in crop production, Wageningen, J. Vos, L. Marcelis, P. de Visser, P. Struik, and J. Evers, Eds., vol. Chapter 5. Springer, 2007, pp. 58-69.

[8] C. Fournier and B. Andrieu, "ADEL-maize: An L-system based model for the integration of growth process from the organ to the canopy. application to regulation of morphogenesis by light availability," Agronomy, vol. 19, pp. 313-327, 1999.

[9] P.-H. Cournède, A. Mathieu, F. Houllier, D. Barthélémy, and P. de Reffye, "Computing competition for light in the GreenLab model of plant growth: a contribution to the study of the effects of density on resource acquisition and architectural development," Annals of Botany, vol. 101, no. 8, 2008.

[10] Y. Ma, M. Wen, Y. Guo, B. Li, P.-H. Cournède, and P. de Reffye, "Parameter optimization and field validation of the functional-structural model greenlab for maize at different population densities," Annals of Botany, vol. 101(8), 2008.

[11] Q. Dong, G. Louarn, Y. Wang, J. Barczi, and P. de Reffye, "Does the structure-fonction model greenlab deal with crop phenotypic plasticity induced by plant spacing? a case study on tomato," Annals of Botany, vol. 101, no. 8, 2008.
[12] S. Lemaire, F. Maupas, P.-H. Cournède, J.-M. Allirand, P. de Reffye, and B. Ney, "Analysis of the density effects on the source-sink dynamics in sugar-beet growth," in Plant growth Modeling, simulation, visualization and their Applications. Proceedings of PMA09, M. Jaeger and B. Li, Eds. IEEE Computer Society (Los Alamitos, California), 2009.

[13] S. Julier, J. Uhlmann, and H. Durrant-Whyte, "A new method for the nonlinear transformation of means and covariances in filters and estimators," IEEE Transactions on Automatic Control, vol. 45, no. 3, pp. 477-482, 2000.

[14] C. Beveridge, J. Weller, S. Singer, and J. Hofer, "Axillary meristem development. budding relationships between networks controlling flowering, branching, and photoperiod responsiveness," Plant Physiol., vol. 131, pp. 927-934, 2003.

[15] S. Lemaire, F. Maupas, P.-H. Cournède, and P. de Reffye, "A morphogenetic crop model for sugar-beet (beta vulgaris l.)." in International Symposium on Crop Modeling and Decision Support: ISCMDS 2008, April 19-22, 2008, Nanjing, China, 2008.

[16] W. Liu, M. Tollenaar, G. Stewart, and W. Deen, "Response of corn grain yield to spatial and temporal variability in emergence," Crop Science, vol. 35, pp. 19-23, 2004.

[17] N. Bosemark, "Genetics and breeding," in Sugar Beet, A. Draycott, Ed. Wiley-Blackwell, 2006, pp. 50-88.

[18] B. Clerget, M. Dingkuhn, E. Gozé, H. Rattunde, and B. Ney, "Variability of phyllochron, plastochron and rate of increase in height in photoperiod-sensitive sorghum varieties," Annals of Botany, 2008.

[19] A. Frank and A. Bauer, "Phyllochron differences in wheat, barley, and forage grasses," Crop Science, vol. 35, pp. 19-23, 1995.

[20] M. Dietze, M. Wolosing, and J. Clark, "Capturing diversity and interspecific variability in allometries: A hierarchical approach," Forest Ecology and Management, vol. 256(11), pp. 1939-1948, 2008.

[21] G. Vieilledent, "Structurer l'incertitude et la variabilité dans les modèles de dynamique forestière," Ph.D. dissertation, AgroParisTech, 2008.

[22] H. Monod, C. Naud, and D. Makowski, "Uncertainty and sensitivity analysis for crop models," in Working with Dynamic Crop Models, D. Wallach, D. Makowski, and J. Jones, Eds. Elsevier, 2006, pp. 55-100.

[23] P. R. Bevington and D. K. Robinson, Data reduction and error analysis for the physical sciences. Boston, MA: McGraw-Hill, 2003.

[24] M. Morgan, M. Henrion, and M. Small, Uncertainty. Cambridge University Press, 1990.

[25] P.-H. Cournède, "Dynamic system of plant growth," HDR Thesis, University of Montpellier II, 2009. 